\title{
Targeted Exploration of Bio-inspired Cascade Reactions: A One-Pot Total Synthesis of Nesteretal A
}

\author{
Axel Leblond, ${ }^{[a]}$ Inès Houari, ${ }^{[a]}$ Karine Leblanc, ${ }^{[a]}$ Erwan Poupon ${ }^{*[a]}$ and Mehdi A. Beniddir ${ }^{\star[a]}$
}

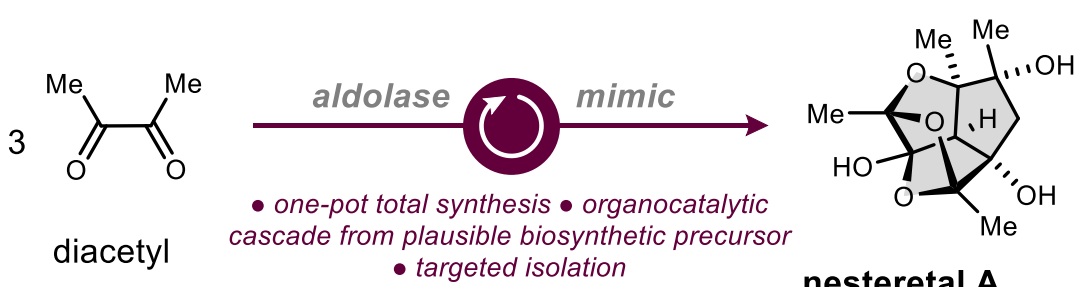

[a] A. Leblond, I. Houari, K. Leblanc, Prof. Dr. E. Poupon, ${ }^{*}$ Dr. M. A. Beniddir*

Chimie des Substances Naturelles

Université Paris-Saclay, CNRS, BioCIS

5, rue Jean-Baptiste Clément, 92296 Châtenay-Malabry, France

E-mail: erwan.poupon@universite-paris-saclay.fr, mehdi.beniddir@universite-paris-saclay.fr

Supporting information for this article is given via a link at the end of the document.

\begin{abstract}
Nesteretal A was recently isolated from a marine actinomycete. An appealing and challenging cage-structure along with an unusual biosynthetic pathway prompted us to explore an expeditious bio-inspired total synthesis of nesteretal A. An unconventional strategy was chosen and a cascade reaction starting from diacetyl was studied. Under organocatalytic conditions mimicking an aldolase-type sequence with a cyclic secondary amine, nesteretal A was detected and targeted through LC-MS/MS and NMR analyses. Starting from a double aldolization of three units, an intramolecular succession of aldolizations and hemiacetalizations probably drives the highly reproducible formation of nesteretal A. An easy isolation protocol has been devised to overcome a non-surprising low yield but largely counterbalanced by the straightforwardness and cost-effectiveness of this first total synthesis of nesteretal A.
\end{abstract}

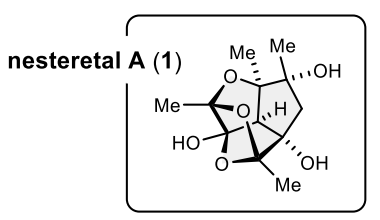

- isolated from Nesterenkonia halobia - interesting biological properties

- chemical challenges: 4 cycles

-6 quaternary carbons

3 tertiary alcohols

Figure 1. Structure of (-)-nesteretal A (1) and synthetic challenges.

(-)-Nesteretal A (1, Figure 1) was isolated by Yang and coll. in 2019 from a culture broth of Nesterenkonia halobia, a scleractinian coral-associated actinomycete of marine origin. ${ }^{[1]}$ Its puzzling cage-like structure, that necessitated impressive structure determination efforts, attracted our attention as well as the biosynthetic pathway, proposed by Yang and coll.,, ${ }^{[1]}$ (2, aka butan2,3-dione, Scheme 1) as a plausible precursor. Indeed, this $\mathrm{C}_{4}$ unit is already known as a microbial metabolite (it provides a buttery aroma to dairy products and some brewed alcohols ${ }^{[2]}$ ) and its biosynthesis is known. ${ }^{[3]}$ This latter implies pyruvate which is converted to acetolactate via a thiamine diphosphate-dependent enzyme (aka thiamine pyrophosphate TPP) which is, in turn, decarboxylated into 2 . It is from diacetyl (2) itself that the hypothesis towards nesteretal A caught our attention. "Retrobiosynthetic" analysis in fact reveals the need for three units of $\mathbf{2}$ and the hypothetic existence of precursor 3 as a central intermediate resulting from a double aldol trimolecular condensation. With its non-symmetrical diol tretraketone motif, 3 logically encodes the requisite structural and reactivity "information" needed for a cascade of intramolecular reactions starting from the formation of the central cyclopentane ring followed by three hemi-acetalizations to build the cage-structure of nesteretal $A$.

As part of a long interest in bio-inspired total synthesis logic starting from simple presumed biosynthetic intermediates, by us $^{[4]}$ and many others, ${ }^{[5]}$ especially relying on "molecular self-assemblies" or "architectural self-construction", ${ }^{[6]}$ we engaged in the total synthesis of nesteretal A (1). We logically questioned if it was possible to fully reproduce the cascade from 2 . The challenge relies on self-assembling in situ three units avoiding, at least partly, oligomerization and hoping for the spontaneous cyclization into $\mathbf{1}$ to act as a driving force in a protecting group-free strategy. ${ }^{[7]}$ This work clearly aims at simplifying to the extreme the quest of the target in total synthesis and analyze at which costs in the context of ideality in total synthesis. ${ }^{[8]}$ 


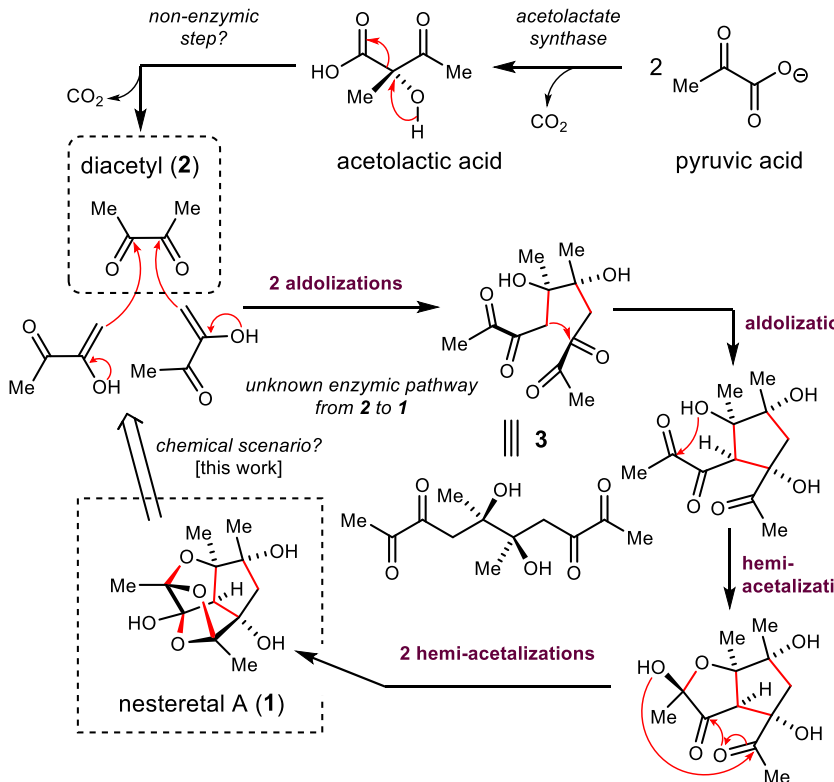

Scheme 1. Biosynthetic hypothesis of nesteretal A (1).

An organocatalytic strategy mimicking plausible enzymic control of the cascade was chosen and (S)-(-)-proline was selected as

An organocatalytic strategy mimicking plausible enzymic control of the cascade was chosen and (S)-(-)proline was selected as one of the most popular catalyst for enamine activation involved in aldol condensations. It is also a simple way to mimic covalent catalysis as found in aldolase-type enzymes (metal-free aldolase of type I to be precise). ${ }^{[9]}$ In that context, performing a challenging condensation of three identical units of low molecular weight has seldomly been studied. Pioneering work from Gijsen and Wong with 2-desoxyribose-4phosphate aldolase (DERA) showed the selfcondensation of acetaldehyde into deoxyhexose. ${ }^{[10]}$ Barbas and coll. using (S)-(-)-proline as a mimic of DERA also demonstrated that a three-unit condensation was possible allowing the formation of hydroxy-hexenals and the formation of two carbon-carbon bonds. ${ }^{[1]}$ In reference to these seminal works on simple substrates and other key-results using (S)-(-)-proline to promote aldol reactions, ${ }^{[12]}$ tetrahydrofuran (THF) and dimethylformamide (DMF) were chosen. Reactions were set up, left at room temperature and monitored for several weeks (see Figure $\mathrm{S} 10$ in Supporting Information). Finding the needle (1) in the haystack was the next challenge (Scheme 2). At first UPLC-HRMS ${ }^{2}$ profiling of the mixture allowed to detect two salient masses at $m / z 257.1031[\mathrm{M}-\mathrm{H}]^{-}$(see Figure $\mathrm{S} 1(\mathrm{~B})$ in Supporting Information). An examination of the MS spectra of these ions exhibited typical water mass loss (see Figure $\mathrm{S} 1(\mathrm{C})$ in Supporting Information). Then, ${ }^{1} \mathrm{H}$ and ${ }^{13} \mathrm{C}$ NMR fingerprinting of the fractions of the crude permitted to efficiently localize nesteretal A spectroscopic features and paved the way towards its isolation (see Figure S8 in Supporting Information, with a global yield estimated at $1 \%$ ). Reactions run in DMF for two weeks appeared to allow the formation of $\mathbf{1}$ in a significant and totally reproducible way (see S9 in
Supporting Information) compared to THF in which no reaction occurred.

Synthetic nesteretal A (1) was fully assigned by NMR and corresponded to natural 1 (see Table S1 and Figure S19 in Supporting Information). Optical rotation was measured $\left(\alpha_{D}=-9\left[c=0.2 ; \mathrm{MeOH} ; 22^{\circ} \mathrm{C}\right]\right.$ versus $\alpha_{D}=-35.7$ $[\mathrm{MeOH}]$ for natural 1). The scalemic character of synthetic 1 was further confirmed by chiral HPLC-ELSD (See Figure $\mathrm{S} 12$ in Supporting Information) and an e.e. of $26 \%$ in favor of natural $(2 R, 4 S, 5 R, 8 R, 9 R, 10 R, 11 R)-\mathbf{1}$ was measured corresponding to a low enantio-induction. Whereas (S)-(-)-proline was found to be indispensable to initiate the cascade (no reaction occurred with 2 in solution in DMF for several weeks, see Figure $S 12$ in Supporting Information), pyrrolidine also leads to similar results providing $( \pm)-\mathbf{1}$ (see Figure $S 11$ in Supporting Information). Moreover, the real implication of these secondary amines beyond the in-situ formation of intermediate 3 is questionable and could be resolved via the conventional synthesis of $\mathbf{3}$. Also, the low enantiodifferentiation opens the way for further studies implying, e.g., a large screening of different organocatalysts for this critical 3-unit-condensation. ${ }^{13]}$

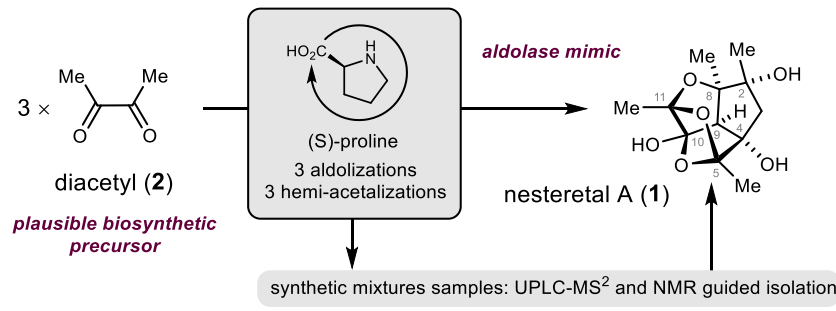

Scheme 2. Cascade reaction and prioritization towards $( \pm)$ nesteretal A (1).

The unusual approach described in this communication permitted to perform the first total synthesis of highly challenging nesteretal $A$ directly from its putative $C_{4}$ precursor. The single operation from costless diacetyl and a secondary amine acting as an organocatalyst permits to obtain - in a probably hardly to compete time and cost - enough material for, biological evaluations. Analyses of our synthesis in the light of atom-, step-, redox- economies can be made and accordingly compete with classical linear multistep-total syntheses. Time will probably come for more rational and stepwise total syntheses of nesteretal $A$ that will allow a full comparison with our strategy. A. They will probably bring further information on the way the cyclization cascade is thermodynamically and stereo-electronically controlled (preliminary $\Delta G$ calculations of aldolization steps were performed by Yang and coll. $\left.{ }^{[1]}\right)$. In the particular case of the organocatalytic strategy described in this paper, it will undoubtedly be an ideal case for optimization. Other compounds being formed in the reaction are currently being characterized and further developments to anticipate chemical diversity and complexity in bioinspired chemistry will be disclosed in due course. At this time anyway, this work also shows the plausibility of the 
biosynthetic hypothesis of nesteretal A (1) from diacetyl (2) and the everlasting interest to ask "how and why" in natural product chemistry ${ }^{[14]}$ and, especially in our case, total synthesis. More precisely in terms of strategy in total synthesis, the striking set of repeating subunits leading to an exotic polycyclic architecture of a natural product can often be considered as an appealing motivation for pursuing its synthesis (condensation of three molecules of $\mathbf{2}$ in the present case). Lessons learned from the literature reveal that while the irresistible temptation of achieving the assembly of complex structures through ambitious cascade oligomerizations of well-chosen biosyntheticallypresumed reactive units can be challenging, ${ }^{[15]}$ a more stepwise approach may ultimately prove successful. ${ }^{[16]}$ The recourse to a synergistic pipeline consisting of LCHRMS/MS and NMR spectroscopic analyses, here applied to chemical synthesis, allowed a successful targeting of the compound.

\section{Acknowledgements}

We thank the French "Agence Nationale pour la Recherche" (grant ANR-19-CE07-0002-01 "ANTIDEPRIM") for funding this study (including $\mathrm{PhD}$ grant of A. L.). Jean-Christophe Jullian and Rémi Franco are thanked for NMR assistance. Dr Bruno Figadère is also acknowledged for fruitful discussion.

Keywords: Nesteretal $\cdot$ bio-inspiration $•$ total synthesis - organocatalysis $\cdot$ cascade reaction

[1] Isolation of nesteretal A: C.-L. Xie, R. Chen, S. Yang, J.-M. Xia, G.-Y. Zhang, C.-H. Zhang, C.-H. Chen, Y. Zhang, X.-W. Yang, Org. Lett. 2019, 21, 8174-8177. For other compounds isolated from this genus (Nesterenkonia flava), see: C.-L. Xie, Q. Liu, J.-M. Xia, Y. Gao, Q. Yang, Z.-Z. Shao, G. Liu, X.-W. Yang, Mar. Drugs 2017, 15, doi:10.3390/md15030071.

[2] (a) S. Clark, C. K. Winter, Comp. Rev. Food Sci. Food Safety 2015, 14, 634-643; (b) K. Krogerus, B. R. Gibson, J. Inst. Brew. 2013, 119, 86-97.

[3] Yang and coll. biosynthetic proposal for the biosynthesis of 2 relies on the first accounts on the topic in the 1960 ies (see: L. F. Chuang, E. B. Collins, J. Bacteriol. 1968, 95, 2083-2089). For updated biosynthetic mechanisms and applications in synthetic biology, see: L. Zhang, Y. Zhang, Q. Liu, L. Meng, M. Hu, M. Lv, K. Li, C. Gao, P. Xu, C. Ma, Sci. Rep. 2015, 5, DOI:10.1038/srep09033 and references cited therein.

[4] Recent examples of bio-inspired cascade reactions from plausible biosynthetic intermediates using protecting groupfree strategies: (a) V. Turpin, M. A. Beniddir, G. Genta-Jouve, A. Skiredj, J.-F. Gallard, K. Leblanc, P. Le Pogam, E. Poupon, Chem. Eur. J. 2020, 26, 12936-12940; (b) S. B. Silva, M. A. Beniddir, J.-F. Gallard, E. Poupon, O. P. Thomas, L. Evanno, Eur. J. Org. Chem. 2019, 5515-5518; (c) S. B. L. Silva, F. Oberhänsli, M.-A. Tribalat, G. Genta-Jouve, J.-L. Teyssié, M.Y. Dechraoui-Bottein, J.-F. Gallard, L. Evanno, E. Poupon, O. P. Thomas, Angew. Chem. Int. Ed. 2019, 58, 520-525; (d) N. Duchemin, A. Skiredj, J. Mansot, K. Leblanc, J.-J. Vasseur, M. A. Beniddir, L. Evanno, E. Poupon, M. Smietana, S. Arseniyadis, Angew. Chem. Int. Ed. 2018, 57, 11786-11791 and references cited therein; (e) D. Lachkar, N. Denizot, G. Bernadat, K. Ahamada, M. A. Beniddir, V. Dumontet, J.-F.
Gallard, R. Guillot, K. Leblanc, E. Otogo N'nang, V. Turpin, C. Kouklovsky, E. Poupon, L. Evanno, G. Vincent, Nat. Chem. 2017, 9, 793-79; (f) S. Benayad, K. Ahamada, G. Lewin, L. Evanno, E. Poupon, Eur. J. Org. Chem. 2016, 1494-1499; (g) S. Benayad, M. A. Beniddir, L. Evanno, E. Poupon, Eur. J. Org. Chem. 2015, 1894-1898; (h) E. Poupon, E. Gravel, Chem. Eur. J. 2015, 21, 10604-10615 and references cited therein.

[5] (a) H. Zhang, A. J. E. Novak, C. S. Jamieson, X. Xue, S. Chen, D. Trauner, K. N. Houk, J. Am. Chem. Soc. 2021, 143, 6601-6608 and reference cited therein; (b) P. D. Brown, A. L. Lawrence, Org. Biomol. Chem. 2019, 17, 1698-1702 (c) M. I. Thomson, G. S. Nichol, A. L. Lawrence, Org. Lett. 2017, 19, 2199-2201; (d) I. De Silvestro, S. L. Drew, G. S. Nichol, F. Duarte, A. L. Lawrence, Angew. Chem. Int. Ed. 2017, 56, 6813-6817 (e) P. D. Brown, A. C. Willis, M. S. Sherburn, A. L. Lawrence, Org. Lett. 2012, 14, 4537-4539.

[6] (a) E. J. Sorensen, Bioorg. Med. Chem. 2003, 11, 32253228; (b) E. Poupon, E. Gravel, Eur. J. Org. Chem. 2008, 2742.

[7] For reviews on protecting group-free synthesis, see among others: (a) R. A. Fernandes, P. Kumar, P. Choudhary, Chem. Commun. 2020, 56, 8569-8590; (b) C. Hui, F. Chen, F. Pu, J. Xu, Nat. Rev. Chem. 2019, 3, 85-107; (c) I. S. Young, P. S. Baran, Nat. Chem. 2019, 1, 193-205.

[8] D. S. Peters, C. R. Pitts, K. S. McClymont, T. P. Stratton, C. Bi, P. S. Baran, Acc. Chem. Res. 2021, 54, 605-617 and key references cited therein.

[9] Aldolase mimic by proline, seminal work: (a) B. List, R. A. Lerner, C. F. Barbas III, J. Am. Chem. Soc. 2000, 122, 23952396; (b) for a review article, see also: H. Gröger, J. Wilken, Angew. Chem. Int. Ed. 2001, 40, 529-532.

[10] H. J. M. Gijsen, C.-H. Wong, J. Am. Chem. Soc. 1994, 116, 8422-8423.

[11] (a) A. Córdova, W. Notz, C. F. Barbas III, J. Org. Chem. 2002, $67,301-303$; (b) using diarylprolinols in place of proline, the outcome was different and resulted in the formation of "only" one carbon-carbon bond from three molecules of acetaldehyde, see: Y. Hayashi, S. Samanta, T. Itoh, H. Ishikawa, Org. Lett. 2008, 10, 5581-5583.

[12] A. B. Northrup, D. W. MacMillan, J. Am. Chem. Soc. 2002, 124, 6798-6799.

[13] L. Albrecht, H. Jiang, K. A. Jørgensen, Angew. Chem. Int. Ed. 2011, 50, 8492-8509.

[14] P. D. Brown, A. L. Lawrence, Nat. Prod. Rep. 2017, 34, 11931202.

[15] Review articles on cascade reactions in the context of total synthesis, see among others: (a) K. C. Nicolaou, D. J. Edmonds, P. G. Bulger, Angew. Chem. Int. Ed. 2006, 45, 7134-7186; (b) K. C. Nicolaou, J. S. Chen, Chem. Soc. Rev. 2009, 38, 2993-3009; (c) C. Grondal, M. Jeanty, D. Enders, Nat. Chem. 2010, 2, 167-178; Y. Jiang, R. E. McNamee, P. J. Smith, A. Sozanschi, Z. Tong, E. A. Anderson, Chem. Soc. Rev. 2021, 50, 58-71.

[16] S. A. Snyder, A. M. El Sohly, F. Kontes, Nat. Prod. Rep. 2011, 28, 897-924. 